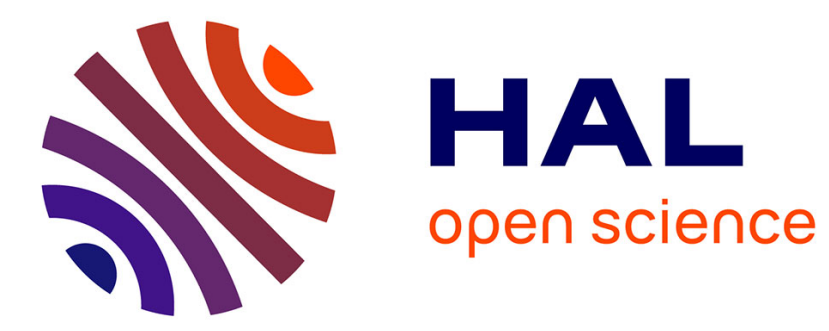

\title{
Deux modèles de population dans un environnement périodique lent ou rapide
}

\author{
Nicolas Bacaër
}

\section{To cite this version:}

Nicolas Bacaër. Deux modèles de population dans un environnement périodique lent ou rapide. Journal of Mathematical Biology, 2020, 80, pp.1021 - 1037. 10.1007/s00285-019-01447-z . hal-02279038v5

\section{HAL Id: hal-02279038 \\ https://hal.science/hal-02279038v5}

Submitted on 19 Jun 2020 (v5), last revised 11 Jan 2023 (v6)

HAL is a multi-disciplinary open access archive for the deposit and dissemination of scientific research documents, whether they are published or not. The documents may come from teaching and research institutions in France or abroad, or from public or private research centers.
L'archive ouverte pluridisciplinaire HAL, est destinée au dépôt et à la diffusion de documents scientifiques de niveau recherche, publiés ou non, émanant des établissements d'enseignement et de recherche français ou étrangers, des laboratoires publics ou privés. 


\title{
Deux modèles de population dans un environnement périodique lent ou rapide
}

\author{
Nicolas Bacaër * \\ J. Math. Biol. 80 (2020) 1021-1037 \\ doi : 10.1007/s00285-019-01447-z \\ hal : 02279038 \\ Traductions : [ar, de, es, it, ja, nl, pt, ru, zh], html]
}

\begin{abstract}
Résumé
On aborde deux problèmes en dynamique des populations dans un environnement périodique lent ou rapide. Dans un premier temps, on obtient un développement limité pour la probabilité de non-extinction d'un processus linéaire de naissance et de mort surcritique à coefficients périodiques lorsque la période est grande ou petite. Si le taux de naissance est inférieur à la mortalité pendant une partie de la période et si la période tend vers l'infini, alors la probabilité de non-extinction tend vers une limite discontinue, liée à un « canard $\gg$ dans un système lent-rapide. Dans un deuxième temps, on étudie un modèle épidémique non linéaire de type S-I-R lorsque le taux de contact oscille rapidement. La taille finale de l'épidémie est proche de celle que l'on obtient en remplaçant le taux de contact par sa moyenne. Une approximation de la correction peut être calculée analytiquement lorsque la reproductivité de l'épidémie est proche de 1 . La correction, qui peut être positive ou négative, est proportionnelle à la fois à la période des oscillations et à la fraction initiale de personnes infectées.
\end{abstract}

\section{Introduction}

Dans un travail publié il y a quelque temps 2, on avait étudié la limite d'un modèle de dynamique des populations, le modèle S-I-S stochastique, dans un environnement périodique lorsque la période tendait vers 0 ou l'infini. On se propose ici d'étudier de la même manière deux modèles en apparence plus simples : un processus linéaire de naissance et de mort et un modèle épidémique déterministe de type S-I-R. Ces deux modèles peuvent être vus comme des approximations d'un même modèle S-I-R stochastique : le processus de naissance

*Institut de recherche pour le développement, Unité de modélisation mathématique et informatique des systèmes complexes, Les Cordeliers, 75006 Paris, France. Courriel : nicolas.bacaer@ird.fr 
et de mort sert d'approximation au début de l'épidémie (les nouvelles infections jouent le rôle des naissances, les guérisons celui des morts); le modèle S-I-R déterministe sert d'approximation quand l'épidémie est déjà bien établie et le nombre de personnes infectées assez grand.

Considérons donc dans un premier temps un processus linéaire de naissance et de mort dans un environnement variable avec un taux de naissance $a(t)$ et une mortalité $b(t)$. La probabilité de non-extinction si l'on part d'un individu au temps $t_{0}$ est

$$
p\left(t_{0}\right)=\frac{1}{1+\int_{t_{0}}^{\infty} b(t) \exp \left[\int_{t_{0}}^{t}[b(s)-a(s)] d s\right] d t},
$$

que l'intégrale au dénominateur soit finie ou infinie [10, p. 220]. Ceci s'applique en particulier au cas où les fonctions $a(t)$ et $b(t)$ sont périodiques de même période, disons $T$. Notons $A(\tau)$ et $B(\tau)$ les fonctions périodiques de période 1 telles que

$$
a(t)=A(t / T), \quad b(t)=B(t / T) .
$$

Considérons les moyennes

$$
\bar{a}=\int_{0}^{1} A(\tau) d \tau, \quad \bar{b}=\int_{0}^{1} B(\tau) d \tau .
$$

Alors $p\left(t_{0}\right)$ est identiquement égal à 1 si $\bar{a} \leq \bar{b}$; c'est une fonction $T$-périodique strictement inférieure à 1 si $\bar{a}>\bar{b}$ (voir [1, §5.2] et [4]). Plaçons nous donc désormais dans le cas surcritique où $\bar{a}>\bar{b}$.

La formule (1) se simplifie lorsque la période $T$ est soit très petite, soit très grande, comme l'a remarqué récemment [7]. Si $t_{0} / T=\tau_{0} \in[0,1]$ est fixé et si $T \rightarrow 0$, alors $p\left(t_{0}\right) \approx 1-\bar{b} / \bar{a}$. Si $t_{0} / T=\tau_{0}$ est fixé et si $T \rightarrow+\infty$, alors $p\left(t_{0}\right) \approx 1-B\left(\tau_{0}\right) / A\left(\tau_{0}\right)$, au moins pour certaines valeurs de $\tau_{0}$ où $A\left(\tau_{0}\right)>B\left(\tau_{0}\right)$.

L'objectif ci-dessous est de préciser ces observations en proposant un développement limité ou un équivalent de la probabilité d'extinction $p\left(t_{0}\right)$. La limite $T \rightarrow 0$ est la plus simple : on a

$$
p\left(t_{0}\right)=\left(1-\frac{\bar{b}}{\bar{a}}\right)\left\{1-\frac{\bar{b} T}{2}+\frac{T}{\bar{a}}\left[\int_{0}^{1} B\left(\tau_{0}+u\right) \int_{\tau_{0}}^{\tau_{0}+u} A(v) d v d u\right]+o(T)\right\} .
$$

Pour l'étude de la limite $T \rightarrow+\infty$, supposons que les fonctions $A(\tau)$ et $B(\tau)$ soient régulières (disons de classe $\mathrm{C}^{1}$ ) et envisageons deux cas :

- ou bien $A(\tau)>B(\tau)$ pour tout $\tau \in[0,1]$ (cas fortement surcritique);

- ou bien $A(\tau)>B(\tau)$ pour tout $\tau \in\left[0, \tau_{1}[\cup] \tau_{2}, 1\right]$ où $0<\tau_{1}<\tau_{2}<1$ et $A(\tau)<B(\tau)$ pour $\tau \in] \tau_{1}, \tau_{2}[$ (cas faiblement surcritique).

Sans perte de généralité, on peut supposer de plus dans le deuxième cas que $\int_{0}^{\tau_{2}}(A(\tau)-B(\tau)) d \tau>0$. Il existe alors un unique $\left.\tau^{*} \in\right] 0, \tau_{1}[$ tel que

$$
\int_{\tau^{*}}^{\tau_{2}}(A(\tau)-B(\tau)) d \tau=0
$$


On montre dans la section 2 que dans le cas fortement surcritique pour tout $\tau_{0} \in[0,1]$ et dans le cas faiblement surcritique pour tout $\tau_{0} \notin\left[\tau^{*}, \tau_{2}\right]$,

$$
p\left(t_{0}\right)=\left(1-\frac{B\left(\tau_{0}\right)}{A\left(\tau_{0}\right)}\right)\left\{1-\frac{A\left(\tau_{0}\right) B^{\prime}\left(\tau_{0}\right)-A^{\prime}\left(\tau_{0}\right) B\left(\tau_{0}\right)}{T A\left(\tau_{0}\right)\left[A\left(\tau_{0}\right)-B\left(\tau_{0}\right)\right]^{2}}+o(1 / T)\right\} .
$$

Dans le cas faiblement surcritique avec $\left.\tau_{0} \in\right] \tau^{*}, \tau_{2}[$,

$$
p\left(t_{0}\right) \sim \frac{\sqrt{2\left[A^{\prime}\left(\tau_{2}\right)-B^{\prime}\left(\tau_{2}\right)\right]}}{B\left(\tau_{0}\right) \sqrt{\pi T}} \mathrm{e}^{T \int_{\tau_{0}}^{\tau_{2}}[A(v)-B(v)] d v} .
$$

Cette dernière probabilité tend exponentiellement vite vers 0 quand $T \rightarrow+\infty$. À la limite, il y a donc une discontinuité en $\tau_{0}=\tau^{*}$. Le fait que la limite soit nulle dans l'intervalle $] \tau^{*}, \tau_{2}$ [ et pas seulement dans l'intervalle $] \tau_{1}, \tau_{2}$ [ est lié à un phénomène de « canard $\gg$ dans un système lent-rapide, comme on l'explique dans la section 2.6

Considérons dans un deuxième temps le modèle épidémique S-I-R déterministe. Soit $N$ la taille supposée constante d'une population, $S(t)$ le nombre de personnes susceptibles d'être infectées au temps $t, I(t)$ le nombre de personnes infectées et $R(t)$ le nombre de personnes retirées de la chaîne de transmission parce qu'elles sont guéries et immunisées. Ainsi $N=S(t)+I(t)+R(t)$. Soit $a(t)$ le taux de contact infectieux et $b$ le taux de guérison. On suppose comme dans le modèle simplifié de Kermack et McKendrick (voir par exemple [11, p. 75]) que

$$
\frac{d S}{d t}=-a(t) \frac{S I}{N}, \quad \frac{d I}{d t}=a(t) \frac{S I}{N}-b I, \quad \frac{d R}{d t}=b I .
$$

Chaque individu susceptible d'être infecté est donc influencé par la proportion $I / N$ d'individus infectés dans la population totale, autrement dit par le $\ll$ champ moyen $\gg$, et non par son voisinage dans une structure de contact particulière.

Dans un travail récent [3], on s'est intéressé à l'influence qu'aurait une oscillation périodique de faible amplitude du taux de contact $a(t)$ sur la taille finale $R(\infty)$ de l'épidémie. On va s'intéresser ici au cas où l'amplitude est quelconque mais où la période des oscillations est petite par rapport à la durée typique de l'épidémie. Pour une épidémie qui durerait quelques semaines, cela représenterait par exemple l'alternance rapide entre le jour et la nuit. Pour une épidémie qui durerait quelques mois, cela représenterait l'alternance entre les jours ouvrés et les fins de semaines, notamment pour les épidémies en milieu scolaire. Pour une épidémie qui durerait plusieurs années voire plusieurs décennies, cela représenterait l'alternance entre les hivers et les étés.

Soit $\varepsilon>0$ la période des oscillations, un paramètre destiné à tendre vers 0 . On suppose que

$$
a(t)=\bar{a}(1+\phi(t / \varepsilon))
$$

avec $\bar{a}>0$ et une fonction $\phi$ continue par morceaux telle que $|\phi(\tau)| \leq 1$ pour tout $\tau$, de sorte que le taux de contact $a(t)$ reste toujours positif ou nul. On suppose de plus que la fonction $\phi$ est périodique de période 1 et de moyenne 
nulle : $\int_{0}^{1} \phi(\tau) d \tau=0$. Ainsi $a(t)$ est une fonction périodique de période $\varepsilon$ et sa moyenne est $\bar{a}$. Prenons comme conditions initiales au début de l'épidémie

$$
S(0)=N-i, \quad I(0)=i, \quad R(0)=0,
$$

avec $0<i<N$.

La section 3.1 montre des simulations de ce modèle. On constate sur des exemples que la taille finale de l'épidémie est remarquablement proche de celle que l'on obtient en remplaçant le taux de contact par sa moyenne. Dans la section 3.2, on propose une explication de cette proximité en faisant quelques hypothèses supplémentaires sur les paramètres du modèle, notamment en supposant que la fraction initiale de personnes infectées est petite et que la reproductivité de l'épidémie reste proche de 1 . On obtient une formule approchée pour la correction à apporter à la taille finale de l'épidémie. Cette correction est proportionnelle à la fois à la période des oscillations et à la fraction initiale de personnes infectées, d'où sa petitesse.

\section{Un processus de naissance et de mort}

\subsection{Calcul préliminaire}

Intéressons-nous tout d'abord au processus linéaire de naissance et de mort. Considérons l'intégrale au dénominateur de la formule (1) et notons-la

$$
J=\int_{t_{0}}^{\infty} b(t) \exp \left[\int_{t_{0}}^{t}[b(s)-a(s)] d s\right] d t .
$$

Par définition, on a

$$
J=\int_{0}^{\infty} B\left(\left(t_{0}+t\right) / T\right) \exp \left[\int_{t_{0}}^{t_{0}+t}[B(s / T)-A(s / T)] d s\right] d t .
$$

Puisque $t_{0} / T=\tau_{0}$, posons $u=t / T$ et $v=s / T$. Alors, en utilisant la périodicité des fonctions $A(\tau)$ et $B(\tau)$, on obtient

$$
\begin{aligned}
J & =T \int_{0}^{\infty} B\left(\tau_{0}+u\right) \exp \left[T \int_{\tau_{0}}^{\tau_{0}+u}[B(v)-A(v)] d v\right] d u \\
& =T \sum_{n=0}^{\infty} \int_{n}^{n+1} B\left(\tau_{0}+u\right) \exp \left[T \int_{\tau_{0}}^{\tau_{0}+u}[B(v)-A(v)] d v\right] d u \\
& =T \sum_{n=0}^{\infty} \int_{0}^{1} B\left(\tau_{0}+u\right) \exp \left[T \int_{\tau_{0}}^{\tau_{0}+u+n}[B(v)-A(v)] d v\right] d u \\
& =T \sum_{n=0}^{\infty} \exp [n T(\bar{b}-\bar{a})] \int_{0}^{1} B\left(\tau_{0}+u\right) \exp \left[T \int_{\tau_{0}}^{\tau_{0}+u}[B(v)-A(v)] d v\right] d u
\end{aligned}
$$

Ainsi

$$
J=\frac{T}{1-\exp [T(\bar{b}-\bar{a})]} \int_{0}^{1} B\left(\tau_{0}+u\right) \exp \left[T \int_{\tau_{0}}^{\tau_{0}+u}[B(v)-A(v)] d v\right] d u
$$




\section{$2.2 \quad$ La limite $T \rightarrow 0$}

Avec le développement limité $\exp (x)=1+x+x^{2} / 2+o\left(x^{2}\right)$ quand $x \rightarrow 0$ dans le facteur devant l'intégrale et plus simplement $\exp (x)=1+x+o(x)$ dans l'intégrale, on obtient

$J=\left(\frac{1}{\bar{a}-\bar{b}}+\frac{T}{2}+o(T)\right)\left(\bar{b}+T\left[\int_{0}^{1} B\left(\tau_{0}+u\right) \int_{\tau_{0}}^{\tau_{0}+u}[B(v)-A(v)] d v d u\right]+o(T)\right)$.

On remarque qu'un terme s'intègre facilement :

$$
\int_{0}^{1} B\left(\tau_{0}+u\right) \int_{\tau_{0}}^{\tau_{0}+u} B(v) d v d u=\frac{1}{2}\left[\left(\int_{\tau_{0}}^{\tau_{0}+u} B(v) d v\right)^{2}\right]_{0}^{1}=\frac{\bar{b}^{2}}{2} .
$$

On en déduit que

$J=\frac{\bar{b}}{\bar{a}-\bar{b}}+\frac{\bar{b} T}{2}+\frac{\bar{b}^{2} T}{2(\bar{a}-\bar{b})}-\frac{T}{\bar{a}-\bar{b}} \int_{0}^{1} B\left(\tau_{0}+u\right) \int_{\tau_{0}}^{\tau_{0}+u} A(v) d v d u+o(T)$.

Puisque $p\left(t_{0}\right)=1 /(1+J)$, on en déduit la formule (2).

\subsection{La limite $T \rightarrow+\infty$ : le cas fortement surcritique}

Reprenons la formule (7). L'intégrale est de la forme

$$
\int_{0}^{1} G(u) \mathrm{e}^{-T F(u)} d u
$$

avec

$$
G(u)=B\left(\tau_{0}+u\right), \quad F(u)=\int_{\tau_{0}}^{\tau_{0}+u}[A(v)-B(v)] d v .
$$

On peut appliquer la méthode de Laplace puisque $T \rightarrow+\infty$. On a

$$
F^{\prime}(u)=A\left(\tau_{0}+u\right)-B\left(\tau_{0}+u\right), \quad F^{\prime \prime}(u)=A^{\prime}\left(\tau_{0}+u\right)-B^{\prime}\left(\tau_{0}+u\right) .
$$

Supposons tout d'abord que $A(\tau)>B(\tau)$ pour tout $\tau \in[0,1]$. Alors $F^{\prime}(u)>$ 0 pour tout $u \in[0,1], F(u)$ a son minimum en $u=0$ et il vaut $F(0)=0$. De plus, $F(u)=\phi_{0} u+\phi_{1} u^{2}+o\left(u^{2}\right)$ quand $u \rightarrow 0$ avec $\phi_{0}=A\left(\tau_{0}\right)-B\left(\tau_{0}\right)$ et $\phi_{1}=\left[A^{\prime}\left(\tau_{0}\right)-B^{\prime}\left(\tau_{0}\right)\right] / 2$. Par ailleurs, $G(u)=\psi_{0}+\psi_{1} u+o(u)$ quand $u \rightarrow 0$ avec $\psi_{0}=B\left(\tau_{0}\right)$ et $\psi_{1}=B^{\prime}\left(\tau_{0}\right)$. D'après un théorème d'Erdélyi [13, p. 85],

$$
\int_{0}^{1} G(u) \mathrm{e}^{-T F(u)} d u=\mathrm{e}^{-T F(0)}\left(\frac{c_{0}}{T}+\frac{c_{1}}{T^{2}}+o\left(\frac{1}{T^{2}}\right)\right)
$$

avec $c_{0}=\psi_{0} / \phi_{0}$ et $c_{1}=\left(\phi_{0} \psi_{1}-2 \phi_{1} \psi_{0}\right) / \phi_{0}^{3}$. Ainsi, puisque $\exp [T(\bar{b}-\bar{a})]$ est exponentiellement petit, la formule (7) donne

$$
J=\frac{B\left(\tau_{0}\right)}{A\left(\tau_{0}\right)-B\left(\tau_{0}\right)}+\frac{A\left(\tau_{0}\right) B^{\prime}\left(\tau_{0}\right)-A^{\prime}\left(\tau_{0}\right) B\left(\tau_{0}\right)}{T\left[A\left(\tau_{0}\right)-B\left(\tau_{0}\right)\right]^{3}}+o(1 / T) .
$$

Avec $p\left(t_{0}\right)=1 /(1+J)$, on en déduit la formule (4). 


\subsection{Le cas faiblement surcritique}

Supposons maintenant qu'il existe $\tau_{1}$ et $\tau_{2}$ tels que $0<\tau_{1}<\tau_{2}<1$ et

$A(\tau)<B(\tau)$ pour tout $\tau \in] \tau_{1}, \tau_{2}[$

$A(\tau)>B(\tau)$ pour tout $\tau \in] 0, \tau_{1}[\cup] \tau_{2}, 1[$.

Comme $\int_{0}^{1}(A(\tau)-B(\tau)) d \tau=\bar{a}-\bar{b}>0$, on a

$$
\int_{0}^{\tau_{2}}(A(\tau)-B(\tau)) d \tau>0 \quad \text { ou } \quad \int_{\tau_{2}}^{1}(A(\tau)-B(\tau)) d \tau>0 .
$$

Quitte à décaler dans le temps les fonctions $A(\tau)$ et $B(\tau)$, supposons que la première inégalité soit vraie.

Il existe alors un unique $\tau^{*} \in\left[0, \tau_{1}\right]$ tel que

$$
\int_{\tau^{*}}^{\tau_{2}}(A(u)-B(u)) d u=0
$$

En effet, notons $h(\tau)$ la fonction définie sur l'intervalle $\left[0, \tau_{1}\right]$ par

$$
h(\tau)=\int_{\tau}^{\tau_{2}}(A(u)-B(u)) d u
$$

Alors $h^{\prime}(\tau)=B(\tau)-A(\tau)<0$ pour $\tau \in\left[0, \tau_{1}\right]$. De plus, $h(0)>0$ d'après la première inégalité (9) et $h\left(\tau_{1}\right)<0$. Il existe donc un unique $\tau^{*} \in\left[0, \tau_{1}\right]$ tel que $h\left(\tau^{*}\right)=0$.

Considérons d'abord le cas où $0<\tau_{0}<\tau_{1}$. La fonction $F(u)$ est croissante pour $u \in\left[0, \tau_{1}-\tau_{0}\right]$, décroisssante pour $u \in\left[\tau_{1}-\tau_{0}, \tau_{2}-\tau_{0}\right]$ et à nouveau croissante pour $u \in\left[\tau_{2}-\tau_{0}, 1\right]$. La fonction $F(u)$ a donc un minimum local en $\tau_{2}-\tau_{0}$. Rappelons que $F(0)=0$.

Si $\left.\tau_{0} \in\right] 0, \tau^{*}\left[\right.$, alors $F\left(\tau_{2}-\tau_{0}\right)>0$. Donc $u=0$ reste le minimum global de $F(u)$ sur l'intervalle [0,1]. Le développement asymptotique (8) reste valide et la formule (4) aussi.

Si en revanche $\tau \in] \tau^{*}, \tau_{1}\left[\right.$, alors $F\left(\tau_{2}-\tau_{0}\right)<0$. Le minimum global de $F(u)$ sur l'intervalle $[0,1]$ est en $u=\tau_{2}-\tau_{0}, F^{\prime}\left(\tau_{2}-\tau_{0}\right)=0, F^{\prime \prime}\left(\tau_{2}-\tau_{0}\right)=$ $A^{\prime}\left(\tau_{2}\right)-B^{\prime}\left(\tau_{2}\right)$ et

$$
\int_{0}^{1} G(u) \mathrm{e}^{-T F(u)} d u \sim \frac{B\left(\tau_{0}\right) \sqrt{\pi}}{\sqrt{2 T\left[A^{\prime}\left(\tau_{2}\right)-B^{\prime}\left(\tau_{2}\right)\right]}} \mathrm{e}^{-T F\left(\tau_{2}-\tau_{0}\right)}
$$

quand $T \rightarrow+\infty$, d'après la méthode de Laplace. Ainsi

$$
J \sim \frac{B\left(\tau_{0}\right) \sqrt{\pi T}}{\sqrt{2\left[A^{\prime}\left(\tau_{2}\right)-B^{\prime}\left(\tau_{2}\right)\right]}} \mathrm{e}^{-T \int_{\tau_{0}}^{\tau_{2}}[A(v)-B(v)] d v}
$$

et $p\left(t_{0}\right)=1 /(1+J) \sim 1 / J$ quand $T \rightarrow+\infty$, ce qui donne la formule (5). 
Considérons maintenant le cas où $\tau_{1}<\tau_{0}<\tau_{2}$. La fonction $F(u)$ est décroissante sur l'intervalle $\left[0, \tau_{2}-\tau_{0}\right]$ puis croisssante sur l'intervalle $\left[\tau_{2}-\tau_{0}, 1\right]$. Son minimum dans l'intervalle $[0,1]$ est donc atteint en $u=\tau_{2}-\tau_{0}$, comme dans le cas précédent. Ainsi, la formule (5) est toujours valable.

Considérons enfin le cas où $\tau_{2}<\tau_{0}<1$. La fonction $F(u)$ est croissante sur l'intervalle $\left[0,1+\tau_{1}-\tau_{0}\right]$, décroisssante sur l'intervalle $\left[1+\tau_{1}-\tau_{0}, 1+\tau_{2}-\tau_{0}\right]$ puis croissante sur l'intervalle $\left[1+\tau_{2}-\tau_{0}, 1\right]$. Elle a donc un minimum local en $1+\tau_{2}-\tau_{0}$ et

$$
F\left(1+\tau_{2}-\tau_{0}\right) \geq \int_{1}^{1+\tau_{2}}(A(\tau)-B(\tau)) d \tau>0
$$

d'après la première inégalité (9). Son minimum global dans l'intervalle $[0,1]$ est donc atteint en $u=0$. Ainsi, c'est la formule (4) qui s'applique.

\subsection{Exemple}

Prenons $B(\tau)=\bar{b}>0$ constant et

$$
A(\tau)=\bar{a}(1+k \cos (2 \pi \tau))
$$

avec $\bar{a}>\bar{b}$ et $0 \leq k \leq 1$. Le cas fortement surcritique correspond à $\bar{a}(1-k)>b$. Si au contraire $\bar{a}(1-k)<b$, alors $\tau_{1}<\tau_{2}$ sont les deux solutions dans l'intervalle $[0,1]$ de l'équation $\cos (2 \pi \tau)=-(1-\bar{b} / \bar{a}) / k$, à savoir

$$
\left.\tau_{1}=\frac{\arccos (-(1-\bar{b} / \bar{a}) / k)}{2 \pi} \in\right] 0,1 / 2\left[, \quad \tau_{2}=1-\tau_{1} .\right.
$$

Le seuil $\tau^{*}$ est la solution dans l'intervalle $\left[0, \tau_{1}\right]$ de l'équation

$$
(\bar{a}-b)\left(\tau_{2}-\tau^{*}\right)+\bar{a} k \frac{\sin \left(2 \pi \tau_{2}\right)-\sin \left(2 \pi \tau^{*}\right)}{2 \pi}=0 .
$$

La formule (2) donne

$$
p\left(t_{0}\right)=\left(1-\frac{\bar{b}}{\bar{a}}\right)\left(1-\frac{\bar{b} k T}{2 \pi} \sin \left(2 \pi \tau_{0}\right)+o(T)\right)
$$

quand $T \rightarrow 0$. Si $\bar{a}(1-k)>b$ ou si $\bar{a}(1-k)<b$ et $\tau_{0} \notin\left[\tau^{*}, \tau_{2}\right]$, alors la formule (4) donne

$$
p\left(t_{0}\right)=\left(1-\frac{\bar{b}}{A\left(\tau_{0}\right)}\right)\left(1-\frac{2 \pi \bar{a} \bar{b} k \sin \left(2 \pi \tau_{0}\right)}{T A\left(\tau_{0}\right)\left[A\left(\tau_{0}\right)-\bar{b}\right]^{2}}+o(1 / T)\right)
$$

quand $T \rightarrow+\infty$. Si $\bar{a}(1-k)<b$ et $\left.\tau_{0} \in\right] \tau^{*}, \tau_{2}[$, La formule (5) donne

$$
p\left(t_{0}\right) \sim \frac{2 \sqrt{-\bar{a} k \sin \left(2 \pi \tau_{2}\right)}}{\bar{b} \sqrt{T}} \exp \left[T(\bar{a}-\bar{b})\left(\tau_{0}-\tau_{2}\right)+\bar{a} k T \frac{\sin \left(2 \pi \tau_{0}\right)-\sin \left(2 \pi \tau_{2}\right)}{2 \pi}\right]
$$

quand $T \rightarrow+\infty$. 
Prenons en particulier $\bar{b}=1, \bar{a}=3$ et $k=0,5$. Alors $\bar{a}(1-k)>\bar{b}$. La figure 1 montre les résultats pour deux valeurs de la période : $T=0,5$ et $T=50$. La probabilité de non-extinction $p\left(t_{0}\right)$, donnée par la formule (11), est estimée par intégration numérique avec le logiciel Scilab. On voit que les formules approchées (2) et (4) donnent de meilleures approximations de $p\left(t_{0}\right)$ que les termes d'ordre 0 . On notera cependant que pour $T \rightarrow+\infty$, l'approximation (4) s'écarte un peu de $p\left(t_{0}\right)$ au voisinage du minimum de $p\left(t_{0}\right)$.

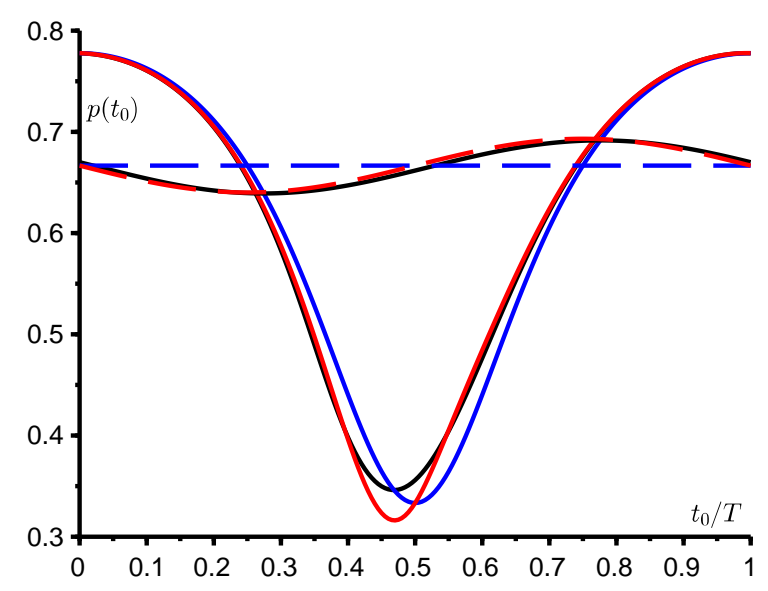

Figure 1 - Deux exemples : $T=0,5$ (pointillés) et $T=50$ (lignes continues). La probabilité de non-extinction $p\left(t_{0}\right)$, donnée par la formule (1), est en noir. En pointillé : la formule approchée (2) en rouge et le terme d'ordre $0,1-\bar{b} / \bar{a}$, en bleu. Lignes continues : la formule approchée (4) en rouge et le terme d'ordre 0 , $1-B\left(\tau_{0}\right) / A\left(\tau_{0}\right)$, en bleu.

Prenons maintenant $\bar{b}=1, \bar{a}=3, k=0,75$ et $T=100$. Alors $\bar{a}(1-k)<\bar{b}$, $\tau^{*} \simeq 0,347, \tau_{1} \simeq 0,424$ et $\tau_{2} \simeq 0,576$. Les diverses formules approchées sont représentées dans la figure 2 , notamment la formule (5) en vert. La probabilité de non-extinction tend vers une limite discontinue, donnée par les courbes bleues pour $\tau<\tau^{*}$ et $\tau>\tau_{2}$, et qui vaut 0 dans l'intervalle $] \tau^{*}, \tau_{2}[$. Il y a un petit problème de raccordement des approximations au niveau de $\tau_{0}=\tau_{2}$, ce qui nous conduit à regarder de plus près ce qui se passe en ce point.

Comme dans le cas où $\tau_{2}<\tau_{0}<1$ de la section 4.2, on voit dans le cas spécial où $\tau_{0}=\tau_{2}$ que $F(u)$ a son maximum global dans $[0,1]$ en $u=0$. Mais cette fois-ci, $F^{\prime}(0)=A\left(\tau_{2}\right)-B\left(\tau_{2}\right)=0$. D'après le même théorème d'Erdélyi [13, p. 85],

$$
J \sim T \int_{0}^{1} G(u) \mathrm{e}^{-T F(u)} d u \sim \frac{B\left(\tau_{2}\right) \sqrt{\pi T}}{\sqrt{2\left[A^{\prime}\left(\tau_{2}\right)-B^{\prime}\left(\tau_{2}\right)\right]}}
$$




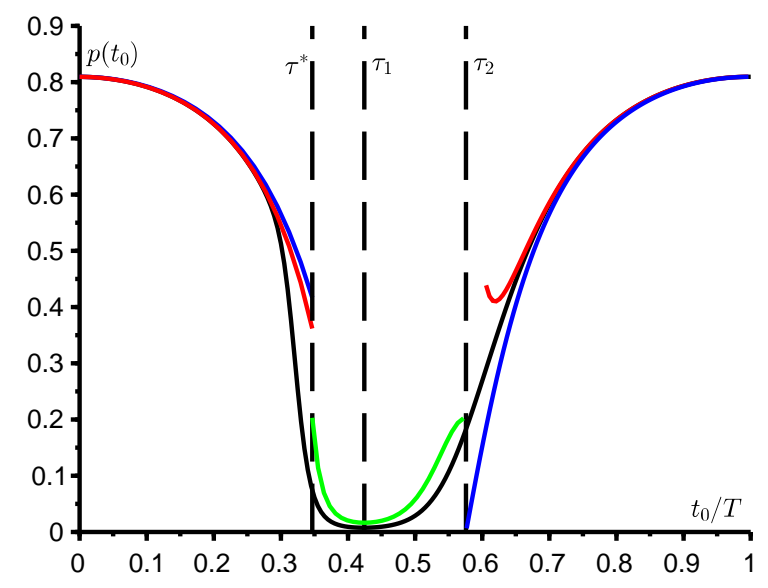

Figure 2 - Comme dans la figure 1 mais avec $T=100$ et $k=0,75$. La formule approchée (5) est en vert.

de sorte que $p\left(\tau_{2} T\right)=1 /(1+J) \sim 1 / J$ quand $T \rightarrow+\infty$. La formule (5) reste valable quand $\tau_{0}=\tau_{2}$. La décroissance exponentielle vers 0 lorsque $\left.\tau_{0} \in\right] \tau^{*}, \tau_{2}$ [ est remplacée par une décroissance en $1 / \sqrt{T}$ au point $\tau_{2}$.

\subsection{Lien avec les « canards »}

La formule (11) pour la probabilité d'extinction $p\left(t_{0}\right)$ au temps $t_{0}$ s'obtient en fait de la manière suivante : si $u>t_{0}$, la probabilité que le processus qui part d'un individu au temps $t_{0}$ soit éteint au temps $u$ est égal à $z\left(u-t_{0}\right)$ avec $z(0)=0$ et

$$
\frac{d z}{d t}=[b(u-t)-a(u-t) z(t)](1-z(t))
$$

dans l'intervalle $t \in\left[0, u-t_{0}\right]$ [4. C'est parce que cette équation de Riccati est résoluble explicitement qu'on obtient la formule (1) pour la probabilité de non-extinction

$$
p\left(t_{0}\right)=1-\lim _{u \rightarrow+\infty} z\left(u-t_{0}\right) .
$$

Prenons par exemple $u=t_{0}+n T$ avec $n$ entier strictement positif. L'équation (10) s'écrit alors

$$
\frac{d z}{d t}=\left[B\left(\frac{t_{0}+n T-t}{T}\right)-A\left(\frac{t_{0}+n T-t}{T}\right) z(t)\right](1-z(t)) .
$$

Posons $s=t / T$ et $z(t)=x(s)$. On a

$$
\frac{d x}{d s}=T\left[B\left(\tau_{0}+n-s\right)-A\left(\tau_{0}+n-s\right) x(s)\right](1-x(s))
$$


sur l'intervalle $s \in[0, n]$. Ceci peut s'écrire comme un système autonome lentrapide :

$$
\begin{aligned}
& \frac{d x}{d s}=T\left[B\left(\tau_{0}+n-y\right)-A\left(\tau_{0}+n-y\right) x(s)\right](1-x(s)), \\
& \frac{d y}{d s}=1
\end{aligned}
$$

pour $s \in[0, n]$, avec $x(0)=0$ et $y(0)=0$. Enfin, $p\left(t_{0}\right)=1-\lim _{n \rightarrow+\infty} x(n)$. Or quand $T \rightarrow \infty$, on voit sur ce système lent-rapide que $x(n) \rightarrow 1$ ou $x(n) \rightarrow$ $B\left(\tau_{0}\right) / A\left(\tau_{0}\right)$. Le fait que $x(n)$ reste sur la branche a priori instable 1 pour $\tau^{*}<\tau_{0}<\tau_{1}$ est donc le même phénomène que ce qui est appelé « canard $\gg$ dans l'étude des systèmes lents-rapides. Rappelons la définition [12, p. 182] :

« Dans un champ lent-rapide de $\mathbb{R}^{2}$, il peut exister des trajectoires qui restent infiniment proches de la courbe lente pendant un temps significatif (non infiniment petit) le long d'un arc attractif, suivi d'un temps significatif passé le long d'un arc répulsif. Une telle trajectoire sappelle [...] un canard. »

[15] a également remarqué l'apparition de pareils canards en lien avec une équation logistique périodique. La relation (3) qui lie $\tau^{*}$ à $\tau_{2}$ est la « relation entrée-sortie $\gg$ correspondante [6, 8].

Ces remarques s'étendent sans doute au cas des processus de naissance et de mort à plusieurs types [4] avec des matrices de naissance $\mathcal{A}(\tau)$ et des matrices de transition ou mort $\mathcal{B}(\tau)$, qui conduisent à un système lent-rapide de la forme

$$
\begin{aligned}
& \frac{d x}{d s}=T\left[\mathcal{B}^{*}\left(\tau_{0}+n-y\right)-\operatorname{diag}(x(s)) \mathcal{A}^{*}\left(\tau_{0}+n-y\right)\right] \text { colonne }(1-x(s)), \\
& \frac{d y}{d s}=1,
\end{aligned}
$$

où les * désignent les matrices transposées. Dans ce cas, la relation entrée-sortie reste à déterminer.

\section{Le modèle S-I-R}

\subsection{Quelques simulations}

Considérons maintenant le modèle S-I-R (6). Les paramètres sont choisis pour être plausibles :

- la population totale est $N=10000$;

- une seule personne est infectée au début de l'épidémie $(i=1)$;

- chaque personne a en moyenne $\bar{a}=15$ contacts par mois ;

- la durée moyenne de l'infection est $1 / b=1 / 10$ mois, soit environ 3 jours ;

- la période $\varepsilon$ est $1 / 4$ de mois, soit environ 7 jours;

- le facteur périodique est

$$
\phi(t / \varepsilon)=k \cos (\omega t+\psi),
$$

où $\omega=2 \pi / \varepsilon$ et $|k| \leq 1$; 
- le déphasage est $\psi=-\pi / 2$ de sorte que $\phi(t / \varepsilon)=k \sin (\omega t)$ et le taux de contact $a(t)$ est dans une phase croissante à $t=0$.

La reproductivité vaut alors $\bar{a} / b=1,5>1$, ce qui garantit le développement d'une épidémie avec une taille finale $R(\infty) \geq N(1-b / \bar{a})[\underline{5}$.

La figure 3 montre deux simulations typiques du modèle : l'une avec $k=0$ (le taux de contact est constant), l'autre avec $k=1$ (le taux de contact oscille). Quoique les courbes pour $k=1$ s'éloignent notablement de celles pour $k=0$ pendant l'épidémie, il est remarquable que les tailles finales $R(\infty)$ dans les deux simulations soient presque indiscernables graphiquement. Ceci sera expliqué dans la section suivante.

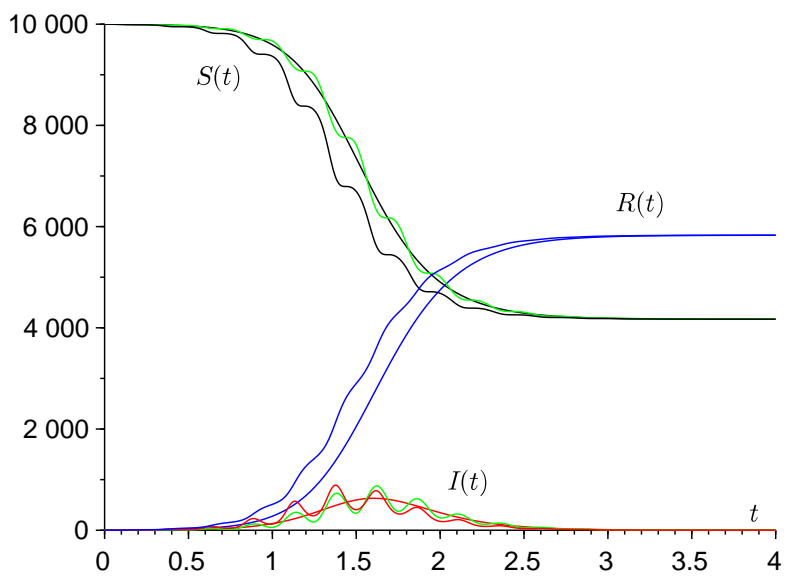

Figure 3 - Simulation d'une épidémie : $S(t)$ en noir, $I(t)$ en rouge, $R(t)$ en bleu. Les courbes non ondulées correspondent à $k=0$, les courbes ondulées à $k=1$. Les courbes vertes sont les approximations du deuxième ordre pour $S(t)$ et $I(t)$.

En réduisant la période des oscillations (par exemple avec $\varepsilon=1 / 8$ de mois), on verrait les courbes $(S(t), I(t), R(t))$ pour $k=1$ conserver leurs oscillations mais se rapprocher de la solution avec $k=0$, que l'on note $(\bar{S}(t), \bar{I}(t), \bar{R}(t))$ car elle correspond à $a(t)=\bar{a}$. C'est d'ailleurs une conséquence du théorème de moyennisation de Fatou [9, théorème 42]. En effet, en posant $\tau=t / \varepsilon$, le système s'écrit

$$
\frac{d S}{d \tau}=-\varepsilon \bar{a}(1+\phi(\tau)) \frac{S I}{N}, \quad \frac{d I}{d \tau}=\varepsilon\left[\bar{a}(1+\phi(\tau)) \frac{S I}{N}-b I\right], \quad \frac{d R}{d \tau}=\varepsilon b I,
$$

avec $\phi(\tau)=\cos (2 \pi \tau+\psi)$. Le théorème assure que, lorsque $\varepsilon \rightarrow 0$,

$$
Z(\tau)-\bar{Z}(\tau):=(S(\tau)-\bar{S}(\tau), I(\tau)-\bar{I}(\tau), R(\tau)-\bar{R}(\tau))=O(\varepsilon)
$$


pendant un temps $\tau$ de l'ordre de $1 / \varepsilon$. Ainsi $Z(t)-\bar{Z}(t)=O(\varepsilon)$ pendant un temps $t$ de l'ordre de 1 . Plus précisément, il existe des constantes $c_{1}, c_{2}, c_{3}$ et $\varepsilon_{0}$ toutes positives telles que pour tout $0<\varepsilon<\varepsilon_{0}$ et tout $t>0$, on ait

$$
\|Z(t)-\bar{Z}(t)\| \leq \varepsilon\left[c_{1} e^{c_{2} t}+c_{3}\right] .
$$

On peut calculer une approximation au second ordre. Écrivons le système (11) sous la forme $d Z / d \tau=\varepsilon f(\tau, Z)$, où $Z=(S, I, R)$ et $f(\tau, Z)$ est $\tau$-périodique de période 1. Alors

$$
\begin{gathered}
f_{0}(Z):=\int_{0}^{1} f(\tau, Z) d \tau=\left(\begin{array}{c}
-\bar{a} S I / N \\
\bar{a} S I / N-b I \\
b I
\end{array}\right), \\
\int_{0}^{\tau}\left[f(\sigma, Z)-f_{0}(Z)\right] d \sigma=\left(\begin{array}{c}
-\bar{a} k \frac{\sin (2 \pi \tau+\psi)-\sin (\psi)}{2 \pi} \frac{S I}{N} \\
\bar{a} k \frac{\sin (2 \pi \tau+\psi)-\sin (\psi)}{2 \pi} \frac{S I}{N} \\
0
\end{array}\right) .
\end{gathered}
$$

Notons qu'il faut retrancher le terme en $\sin (\psi)$ pour que ces dernières fonctions soient de moyenne nulle. D'après [9, théorème 44], on a

$$
\begin{aligned}
& S(\tau)=\bar{S}(\tau)-\varepsilon \frac{\bar{a} k \sin (2 \pi \tau+\psi)}{2 \pi} \frac{\bar{S}(\tau) \bar{I}(\tau)}{N}+O\left(\varepsilon^{2}\right), \\
& I(\tau)=\bar{I}(\tau)+\varepsilon \frac{\bar{a} k \sin (2 \pi \tau+\psi)}{2 \pi} \frac{\bar{S}(\tau) \bar{I}(\tau)}{N}+O\left(\varepsilon^{2}\right)
\end{aligned}
$$

et $R(\tau)=\bar{R}(\tau)+O\left(\varepsilon^{2}\right)$ sur un intervalle de temps $\tau$ de l'ordre de $1 / \varepsilon$. Autrement dit,

$$
\begin{aligned}
S(t) & =\bar{S}(t)-\frac{\bar{a} k \sin (\omega t+\psi)}{\omega} \frac{\bar{S}(t) \bar{I}(t)}{N}+O\left(1 / \omega^{2}\right), \\
I(t) & =\bar{I}(t)+\frac{\bar{a} k \sin (\omega t+\psi)}{\omega} \frac{\bar{S}(t) \bar{I}(t)}{N}+O\left(1 / \omega^{2}\right)
\end{aligned}
$$

et $R(t)=\bar{R}(t)+O\left(1 / \omega^{2}\right)$ sur un intervalle de temps $t$ de l'ordre de 1 . Ces approximations de $S(t)$ et $I(t)$ sont représentées en vert dans la figure 3 .

Remarquons qu'avec une période du taux de contact qui est petite, on n'observe pas de courbe épidémique avec plusieurs grandes vagues, contrairement aux simulations de [5]. C'est que le système se rapproche de plus en plus du cas où le taux de contact est moyenné, qui ne donne qu'une seule vague épidémique.

\subsection{Proximité des tailles finales}

En écrivant la première équation (6) sous la forme $\frac{d}{d t}(\log S)=-a(t) I / N$, en intégrant entre $t=0$ et $t=+\infty$, en tenant compte des conditions initiales et de ce que $\int_{0}^{\infty} I(t) d t=R(\infty) / b$, on trouve facilement comme dans [3] que

$$
\log \frac{N-R(\infty)}{N-i}+\frac{\bar{a}}{b} \frac{R(\infty)}{N}+\frac{\bar{a}}{N} \int_{0}^{\infty} I(t) \phi(t / \varepsilon) d t=0 .
$$


L'intégrale oscillante $\int_{0}^{\infty} I(t) \phi(t / \varepsilon) d t$ tend sans doute vers 0 quand $\varepsilon \rightarrow 0$. En effet, on sait d'une part que $I(t) \simeq \bar{I}(t)$. D'autre part, du moins lorsque $\phi$ est un cosinus, l'intégrale $\int_{0}^{\infty} \bar{I}(t) \phi(t / \varepsilon) d t$ tend vers 0 quand $\varepsilon \rightarrow 0$. C'est une conséquence du fait que $\bar{I}(t)$ soit une fonction positive intégrable, car $\int_{0}^{\infty} \bar{I}(t) d t=\bar{R}(\infty) / b$, et du fait que la transformée de Fourier d'une fonction intégrable tende vers 0 à l'infini.

Il en résulte que $R(\infty) \rightarrow \bar{R}(\infty)$ quand $\varepsilon \rightarrow 0$. La question est de savoir à quelle vitesse ceci se produit. En première approximation, un développement limité de l'équation (12) comme dans [3] donne

$$
R(\infty) \simeq \bar{R}(\infty)+\frac{\bar{a}}{N /(N-\bar{R}(\infty))-\bar{a} / b} \int_{0}^{\infty} \bar{I}(t) \phi(t / \varepsilon) d t
$$

Puis on utilise pour $\bar{I}(t)$ l'expression analytique approchée en forme de cloche symétrique obtenue par Kermack et McKendrick (voir par exemple [3] ou [10]), ce qui suppose que la reproductivité $\bar{a} / b$ reste proche de 1 tout en étant supérieure à 1 et que la fraction initiale infectée $i / N$ soit petite $(i / N \ll 1)$ :

$$
\bar{I}(t) \simeq \frac{N X}{\operatorname{ch}^{2}[Y(t-T)]},
$$

où $\operatorname{ch}(\cdot)$ désigne le cosinus hyperbolique tandis que $X, Y$ et $T$ vérifient, sous l'hypothèse supplémentaire vraisemblable $i / N \ll(\bar{a} / b-1)^{2}$ (la fraction initiale infectée est bien plus petite que la reproductivité n'est proche de 1),

$$
X \simeq \frac{(1-b / \bar{a})^{2}}{2}, \quad Y \simeq \frac{\bar{a}-b}{2}, \quad T \simeq \frac{\log \left[2(N / i)(1-b / \bar{a})^{2}\right]}{\bar{a}-b} .
$$

Notons que $T$ est une approximation du temps qui s'écoule avant le pic de l'épidémie dans un environnement constant.

Supposons enfin que $\phi(\tau)=k \cos (2 \pi \tau+\psi)$ comme dans la figure 3. Notons $\operatorname{Re}(\cdot)$ la partie réelle d'un nombre complexe et i le nombre imaginaire habituel (à ne pas confondre avec $i$, la population infectée initialement). On a alors, en utilisant un résultat classique sur le calcul asymptotique des intégrales complexes avec une phase qui n'est pas stationnaire de sorte que le terme principal vient du bord de l'intervalle d'intégration [14, théorème 3],

$$
\begin{aligned}
\int_{0}^{\infty} \bar{I}(t) \phi(t / \varepsilon) d t & \simeq N X k \int_{0}^{\infty} \frac{\cos (\omega t+\psi)}{\operatorname{ch}^{2}[Y(t-T)]} d t \\
& =N X k \operatorname{Re}\left(\mathrm{e}^{\mathrm{i} \psi} \int_{0}^{\infty} \frac{\mathrm{e}^{\mathrm{i} \omega t}}{\operatorname{ch}^{2}[Y(t-T)]} d t\right) \\
& \simeq-N X k \operatorname{Re}\left(\frac{\mathrm{e}^{\mathrm{i} \psi}}{\mathrm{i} \omega \operatorname{ch}^{2}(-Y T)}\right) \\
& =-\frac{N X k \sin (\psi)}{\omega \operatorname{ch}^{2}(Y T)}
\end{aligned}
$$


Avec les approximations (14), on voit en plus que

$$
\operatorname{ch}^{2}(Y T) \simeq \mathrm{e}^{2 Y T} / 4 \simeq(N / i)(1-b / \bar{a})^{2} / 2 \simeq(N / i) X,
$$

ce qui donne finalement pour $\omega \rightarrow+\infty$

$$
R(\infty) \simeq \bar{R}(\infty)-\frac{\bar{a} k \sin (\psi)}{N /(N-\bar{R}(\infty))-\bar{a} / b} \frac{i}{\omega} .
$$

La taille finale $\bar{R}(\infty)$ dans un environnement constant est l'unique solution strictement positive de l'équation

$$
1-\frac{\bar{R}(\infty)}{N}=(1-i / N) \exp \left(-\frac{\bar{a}}{b} \frac{\bar{R}(\infty)}{N}\right)
$$

ce que l'on retrouve facilement à partir de l'équation (12). Puisque $i \ll N$, la taille finale $\bar{R}(\infty)$ ne dépend que très peu de la condition initiale $i$. Elle est donnée de manière approchée par la solution strictement positive de

$$
1-\frac{\bar{R}(\infty)}{N} \simeq \exp \left(-\frac{\bar{a}}{b} \frac{\bar{R}(\infty)}{N}\right) .
$$

Le terme correcteur dans l'équation (15), qui peut être positif ou négatif selon le signe de $\sin (\psi)$, est donc à la fois proportionnel à $1 / \omega$, c'est-à-dire à la période $\varepsilon$ qui est petite, et à la fraction $i / N$ de personnes initialement infectées, qui est également petite. C'est pourquoi, comme annoncé, la taille finale de l'épidémie est remarquablement proche de celle que l'on obtient en remplaçant le taux de contact par sa moyenne.

Ceci est illustré dans la figure 4 avec des valeurs des paramètres identiques à celles de la figure 3 pour $k=1$. On a fait varier la période $\varepsilon$. On a aussi essayé deux conditions initiales : $i=1$ et $i=2$. Lorsque $\varepsilon \rightarrow 0$, la courbe pour $R(\infty)$ semble bien tangente à l'approximation (15). On remarquera sur l'échelle verticale la petitesse de la différence relative $[R(\infty)-\bar{R}(\infty)] / \bar{R}(\infty)$. Comme $N=10000$, cela se traduit pour la taille finale de l'épidémie au maximum par une différence de 1 ou 2 personnes (la taille devrait en principe être un nombre entier). Notons que la reproductivité $\bar{a} / b$ vaut ici 1,5 , de sorte que l'approximation (13) de Kermack et McKendrick est encore relativement bonne [10, p. 240].

Si $\psi$ est nul ou un multiple entier du nombre $\pi$, le terme correcteur dans l'équation (15) est nul. Mais comme il s'agit d'un cas exceptionnel, il ne vaut peut-être pas la peine de trouver un nouvel équivalent pour l'intégrale $\int_{0}^{\infty} \bar{I}(t) \phi(t / \varepsilon) d t$ ci-dessus.

En conclusion, on peut dire que la proximité des tailles finales $R(\infty)$ et $\bar{R}(\infty)$ justifie d'une certaine manière le fait que dans beaucoup de modèles épidémiques, on néglige les oscillations de courte période pour ne considérer que des taux de contacts moyens. 


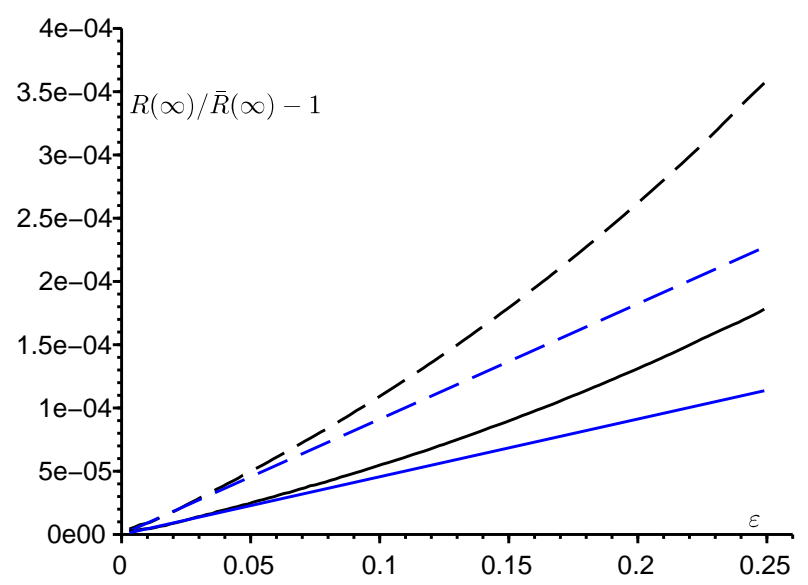

FIGURE 4 - La différence relative $[R(\infty)-\bar{R}(\infty)] / \bar{R}(\infty)$ entre les tailles finales des épidémies en fonction de la période $\varepsilon$. En noir, $R(\infty)$ est estimé en simulant le système d'équations différentielles. L'approximation (15) est représentée en bleu. Les paramètres sont les mêmes que dans la figure 3 avec $k=1$, sauf que la période $\varepsilon$ varie entre 0 et 0,25 mois et que $i=1$ (lignes continues) ou $i=2$ (pointillés). 


\section{Références}

[1] N. Bacaër, Approximation of the basic reproduction number $R_{0}$ for vectorborne diseases with a periodic vector population, Bull. Math. Biol. 69 (2007) 1067-1091.

[2] N. Bacaër, On the stochastic SIS epidemic model in a periodic environment, J. Math. Biol. 71 (2015) 491-511.

[3] N. Bacaër, Sur la taille finale des épidémies dans un environnement périodique, C. R. Biologies 342 (2019) 119-123.

[4] N. Bacaër, E.H. Ait Dads, On the probability of extinction in a periodic environment, J. Math. Biol. 68 (2014) 533-548.

[5] N. Bacaër, M.G.M. Gomes, On the final size of epidemics with seasonality, Bull. Math. Biol. 71 (2009) 1954-1966.

[6] E. Benoît, Relation entrée-sortie, C. R. Acad. Sci. Paris (série I) 293 (1981) 293-296.

[7] Ph. Carmona, Pathogen emergence in seasonal environments (2019) https://www.math.sciences.univ-nantes.fr/ carmona/pages/pathemslides.pdf, www.biorxiv.org/cgi/content/short/753442v1

[8] P. De Maesschalck, S. Schecter, The entryexit function and geometric singular perturbation theory, J. Diff. Eq. 260 (2016) 6697-6715.

[9] J.-P. Françoise, Oscillations en biologie, Springer/SMAI, Berlin, 2005.

[10] J. Gani, Processus stochastiques de population, in : P.-L. Hennequin (éd.), École d'été de probabilités de Saint-Flour IV-1974, Springer, Berlin, 1975, p. 188-293.

[11] A. Hillion, Les Théories mathématiques des populations, Presses Universitaires de France, Paris, 1986.

[12] C. Lobry, La Relation ressource-consommateur - modélisation mathématique, ISTE Editions, Londres, 2018.

[13] F.W.J. Olver, Asymptotics and Special Functions, Academic Press, New York, 1974.

[14] J.-L. Ovaert, J.-L. Verley, Calculs asymptotiques, in : Dictionnaire des mathématiques - algèbre, analyse, géométrie, Albin Michel, Paris, 1997, p. $47-62$.

[15] F. Verhulst, The hunt for canards in population dynamics : A predator-prey system, Int. J. Nonlin. Mech. 67 (2014) 371-377. 